LỰA CHỌN NHÀ CUNG CẤP TRONG QUẢN LÝ CHUỖI CUNG ÚNG: CÁCH TIẾP CẬN AHP. NGHIÊN CÚU CHỌN NHÀ CUNG CẤP CARTON CHO TRƯờnG HỢP CÔNG TY PVM

\title{
SUPPLIER SELECTION IN SUPPLY CHAIN MANAGEMENT: AHP APPROACH. A CASE STUDY INTHE PVM COMPANY'S CARTON SUPPLIER SELECTION
}

\author{
Nguyễn Thị Đức Nguyên ${ }^{1}$ \\ Lê Phước Luông ${ }^{1}$ \\ Lê Hoàng Lan ${ }^{2}$ \\ ${ }^{1}$ Khoa quản lý công nghiệp, Truờng đại học Bách Khoa, ĐHQG-HCM \\ ${ }^{2}$ Học viên cao hoc khoa quản lý công nghiệp.
}

\begin{abstract}
TÓM TĂT
Nghiên cưu này nhằm: (1) tổng kết các tiêu chí lựa chọn nhà cung cấp trong quản lý chuỗi cung ưng về mặt lý thuyết và xây dựng các tiêu chí lựa chọn nhà cung úng carton cho công ty PVM đáp ứng thị truờng xuất khẩu; và (2) phân tích, so sánh giữa phuoong án lựa chọn mới nhà cung ứng với phuơng án phát triển nhà cung ứng hiện tại. Phuoong pháp phỏng vấn sâu chuyên gia và phuong pháp AHP với hỗ trọ của phần mềm Expert Choice để sủ dụng để thu thập dũ liệu, xủ lý dũ liệu, xây dụng các tiêu chí đánh giá và lựa chọn phuoong án thay thế. Kết quả của nghiên cứu cho thấy có 6 tiêu chí chính quan trọng trong việc lụa chọn nhà cung cấp carton đối với công ty PVM bao gồm nhu sau: chất lự̛ng, chi phí, giao hàng, độ tin cây, tài chính, dịch vụ. Thông qua khảo sát, có 2 nhà cung cấp được đura vào nghiên cứu đánh giá, công ty BH và công ty TA. Kết quả phân tích cuối cùng đề xuất công ty PVM nên lụa chọn công ty BH làm nhà cung cấp carton cho thị truờng xuất khẩu.
\end{abstract}

Tù khóa: Analytic Hierarchy Process (AHP); Lựa chọn nhà cung cấp; Quản lý chuỗi cung ứng

\begin{abstract}
This study aims to: (1) summarize the criteria for selecting suppliers in the supply chain management from literature review and apply of the found criteria to construct the criteria for selecting carton suppliers for PVM Company expanding export market, and (2) analyze and comparethe strategy offinding the new supplier or developing the existing supplier. The in-depth interviews with experts in supply chain management and AHP approach with the support of the Expert Choice software are used for data collection, data processing, evaluating and selecting supplier alternatives.
\end{abstract}

\section{Trang 134}


The results show that there are 6 main significantcriteria in choosing carton suppliers for the PVM Company including: quality, cost, delivery, reliability, financial, service. Also, by this study, there are two suppliers, such as, BH Companyand TA Company. Finally, the analytical results suggest PVM Companyshould choose the BH Company as carton supplier for the PVM Company's export market development.

Keyword: Analytic Hierarchy Process (AHP); Supplier Selection;Supply Chain Management.

\section{GIỚI THIỆU}

Ngày nay, tập trung vào chuỗi cung ứng là một trong những chiến lược để tạo lợi thế cạnh tranh trong thị trường toàn cầu hóa. Lựa chọn nhà cung cấp lại là việc quan trọng đối với doanh nghiệp liên quan đến tối ưu hóa chuỗi cung ứng để giảm thiểu chi phí và tăng khả năng đáp ứng kịp thời với yêu cầu thay đổi liên tục của khách hàng. Trước đây, việc lựa chọn nhà cung ứng theo phương pháp truyền thống dựa trên tiêu chí giá cả [4]. Tuy nhiên, doanh nghiệp nhận ra mỗi tiêu chí giá cả thì không hiệu quả khi lựa chọn nhà cung cấp. Các nghiên cứu và công ty chuyển qua phương pháp lựa chọn dựa trên đa tiêu chí liên quan môi trường, xã hội, chính trị và sự hài lòng khách hàng, bên cạnh những vấn đề truyền thống như chất lượng, giao hàng, chi phí và dịch vụ $[4 ; 5 ; 6 ; 11]$.

Trước tình hình trên, công ty PVM, chuyên sản xuất kẹo và chewing gum cao cấp cho thị trường trong nước và nhiều quốc gia khác nhau, cũng không là một ngoại lệ. Đầu năm 2014, công ty PVM nhận phàn nàn của khách hàng nước ngoài về sự cố chất lượng của thùng carton. Công ty truy tìm nhà cung cấp carton hiện tại gây lỗi, cung cấp carton không đủ chất lượng, đánh giá lại nhà cung cấp; và sau đó, bộ phận mua hàng chọn nhà cung cấp VT vì chất lượng tốt hơn cho các thị trường xuất khẩu khó tính. Từ đó, nhiều sản phẩm carton mới của công ty PVM được giao cho VT cung cấp. Đến cuối năm 2014, với nhu cầu carton của công ty PVM ngày càng nhiều, VT thường xuyên không đáp ứng kịp nhu cầu đơn hàng PVM yêu cầu. Trước vấn đề tồn tại, giám đốc chuỗi cung ứng công ty PVM quyết định nghiên cứu, tìm kiếm thêm nhà cung cấp carton mới cho thị trường xuất khẩu mà nhà cung ứng đó phải phù hợp và thích ứng nhanh với tình hình hiện tại của công PVM. Hiện có hai phương án mà công ty PVM xem xét: $(a)$ Tìm kiếm một nhà cung cấp carton hoàn toàn mới; và $(b)$ Phát triển nhà cung cấp $\mathrm{BH}$, vốn đang cung cấp carton cho thị trường nội địa, để họ cung cấp thêm hàng cho thị trường xuất khẩu. Vấn đề cần đặt ra của công ty PVM liên quan đến bài toán ra quyết định đa tiêu chí. Để hỗ trợ cho quá trình ra quyết định này, phương pháp AHP là một trong các phương pháp định lượng có cấu trúc và phân tích các quyết định phức tạp, giúp cho doanh nghiệp đưa ra quyết định tốt hơn [14].

\section{CƠ SỞ LÝ THUYÊT VÀ PHƯƠNG PHÁP NGHIÊN CÚU}

\subsection{Các phương pháp liên quan bài toán ra quyết định đa tiêu chí}

Lựa chọn nhà cung cấp trong quản lý chuỗi cung ứng bản chất liên quan đến bài toán ra quyết định đa tiêu chí (MCDM). Các nghiên cứu về MCDM phân ra thành hai loại chính: ra quyết định đa mục tiêu (MODM) và ra quyết định đa thuộc tính (MADM). MODM nhắm vào các loại 
bài toán có không gian quyết định liên tục, chứa một tập lớn các phương án [9]. MADM thích hợp với các loại bài toán với không gian ra quyết định rời rạc, có các phương án được xác định trước; và thường được sử dụng trong việc lựa chọn tập phương án ra quyết định tốt nhất từ danh sách hữu hạn các phương án sẵn có (Bảng 1) [9].

Bảng 1: Tóm tắt các phương pháp được sử dụng trong bài toán MADM

\begin{tabular}{|c|c|c|c|}
\hline $\begin{array}{l}\text { Phương } \\
\text { pháp }\end{array}$ & Mô tả & Uu điểm & Nhược điểm \\
\hline AHP & $\begin{array}{l}\text { - So sánh cặp giữa các lựa } \\
\text { chọn thay thế với các tiêu } \\
\text { chí khác nhau và ước tính } \\
\text { trọng số của các tiêu chí. }\end{array}$ & $\begin{array}{l}\text { - Dễ sử dụng, khả năng mở rộng } \\
\text { dễ dàng. } \\
\text { - Linh động, trực quan và nhất } \\
\text { quán. } \\
\text { - Vấn đề được xây dựng thành một } \\
\text { cấu trúc phân cấp, mức độ quan } \\
\text { trọng của mỗi yếu tố rõ ràng, dế } \\
\text { điều chỉnh phù hợp với kích thước } \\
\text { nhiều vấn đề. }\end{array}$ & $\begin{array}{l}\text { - Không có quy tắc trong xếp } \\
\text { hạng, do đó có thể dẫn đến } \\
\text { mâu thuẫn giữa việc phán xét } \\
\text { và sắp xếp tiêu chí. }\end{array}$ \\
\hline ELECTRE & $\begin{array}{l}\text { - Được sử dụng để chọn } \\
\text { giải pháp tốt nhất với thuận } \\
\text { lợi tối đa và ít xung đột với } \\
\text { chức năng của các tiêu chí } \\
\text { khác. }\end{array}$ & $\begin{array}{l}\text { - Thứ tự xếp hạng ưu tiên hơn } \\
\text { được sử dụng }\end{array}$ & - Tốn thời gian \\
\hline TOPSIS & $\begin{array}{l}\text { - Dùng để xác định một sự } \\
\text { thay thế mà gần với giải } \\
\text { pháp lý tưởng nhất và xa } \\
\text { giải pháp tiêu cực nhất } \\
\text { trong một không gian toán } \\
\text { đa chiều. }\end{array}$ & $\begin{array}{l}\text { - Có quy trình đơn giản. } \\
\text { - Dễ sử dụng và lập trình. } \\
\text { - Số lượng các bước là giống nhau } \\
\text { bất kế số lượng các thuộc tính. }\end{array}$ & $\begin{array}{l}\text { - Việc sử dụng ma trận } \\
\text { khoảng cách không xem xét } \\
\text { mối tương quan giữa các } \\
\text { thuộc tính.. } \\
\text { - Khó khăn trong việc đo và } \\
\text { giữ tính nhất quán của } \\
\text { phương án. }\end{array}$ \\
\hline $\begin{array}{l}\text { PROMET } \\
\text { HEE }\end{array}$ & & $\begin{array}{l}\text { - Dễ sử dụng; không yêu cầu giả } \\
\text { định rằng các tiêu chí là tương } \\
\text { xứng. }\end{array}$ & $\begin{array}{l}\text { - Không cung cấp một } \\
\text { phương pháp rõ ràng để gán } \\
\text { trọng số. }\end{array}$ \\
\hline $\begin{array}{l}\text { Grey } \\
\text { theory }\end{array}$ & $\begin{array}{l}\text { - Xử lý tất cả trường hợp } \\
\text { dữ liệu không hoàn chỉnh } \\
\text { và khắc phục những khuyết } \\
\text { điểm của các phương pháp } \\
\text { khác. }\end{array}$ & $\begin{array}{l}\text { - Thông tin hoàn hảo có một giải } \\
\text { pháp duy nhất. }\end{array}$ & $\begin{array}{l}\text { - Không cung cấp giải pháp } \\
\text { tối ưu. }\end{array}$ \\
\hline
\end{tabular}

Từ Bảng 1 so sánh ưu và nhược điểm của các phương pháp ra quyết định, phương pháp AHP là một trong số những kỹ thuật ra quyết định được áp dụng khi lựa chọn nhà cung cấp giúp giải quyết vấn đề với những tiêu chí xung đột, khác biệt lẫn nhau. Nhiều nghiên cứu áp dụng AHP để giải quyết vấn đề ở các khía cạnh khác nhau, ví dụ: lựa chọn nhà cung cấp cho nhà máy sợi Polyamide [8], nhà máy dược phẩm ở Ghana [4], nhà máy thép [6], cho ngành công nghiệp quy mô nhỏ, quy mô trung bình và quy mô lớn [5; 11], ... Vài khoá luận thạc sỹ tại Việt Nam gần đây áp dụng AHP để giải quyết bài toán lựa chọn nhà cung cấp ở lĩnh vực may [10], thiết bị di động viễn thông [13]...Tuy nhiên, các nghiên cứu này vẫn chưa lý luận rõ ràng cách tiếp cận $\mathrm{AHP}$ và các tiêu chí phù hợp để đánh giá lựa chọn nhà cung cấp trong quản lý chuỗi cung ứng về mặt lý thuyết và thực tiễn. Thêm vào đó, rất ít các nghiên cứu áp dụng $\mathrm{AHP}$ vào lĩnh vực

\section{Trang 136}


sản xuất thực phẩm, lĩnh vực liên quan đến vấn đề an toàn, sức khoẻ. Vì vậy, AHP được chọn để đánh giá lựa chọn nhà cung cấp carton cho công ty PVM trong nghiên cứu này.

\subsection{Phuơng pháp AHP}

Phương pháp AHP được phát minh năm 1980 bởi Saaty, một phương pháp ra quyết định theo phân tích thứ bậc, một phương pháp tính toán đơn giản, có cơ sở lý thuyết vững chắc nhằm hỗ trợ các cá nhân hay nhóm chuyên gia đánh giá, phân tích và ra quyết định lựa chọn các phương án cho trước hay xử lý các vấn đề ra quyết định đa thuộc tính [14]. AHP dùng để sắp xếp các phương án quyết định và chọn một phương án thỏa mãn các tiêu chí cho trước; giúp trả lời các câu hỏi như "nên chọn phương án nào" hay "phương án nào tốt nhất" bằng cách chọn một phương án tốt nhất thỏa mãn các tiêu chí của người ra quyết định [14].

AHP có 3 phân đoạn cơ bản: phân giải vấn đề cần giải quyết thành các thứ bậc, so sánh sự đánh giá của những phần tử theo cách so sánh cặp giữa các yếu tố và tổng hợp độ ưu tiên bằng cách xác định ma trận trọng số [7]. Trên cơ sở trên, các bước phân tích AHP theo sau [14]: (1) phân tích vấn đề và xác định lời giải yêu cầu; (2) xác định các yếu tố và xây dựng cây phân cấp yếu tố; (3) điều tra thu thập ý kiến chuyên gia về mức độ ưu tiên; (4) thiết lập các ma trận so sánh cặp; (5) tính toán trọng số cho từng mức, từng nhóm yếu tố; (6) tính tỷ số nhất quán (CR). Tỷ số $\mathrm{CR}$ phải nhỏ hơn hay bằng $10 \%$, nếu lớn hơn, cần thực hiện lại bước 3,4,5; (7) thực hiện bước $3,4,5,6$ cho tất cả các mức và các nhóm yếu tố trong cây phân cấp; và (8) tính toán trọng số tổng hợp và nhận xét.

\subsection{Phuơng pháp nghiên cúu}

Nghiên cứu này nhằm trả lời hai câu hỏi nghiên cứu sau: (a) Về mặt lý thuyết quản lý chuỗi cung ứng, phương pháp và những tiêu chí phù hợp cần được quan tâm khi lựa chọn nhà cung cấp; và ( $b$ ) Với trường hợp cụ thể công ty thực phẩm PVM, công ty nên áp dụng lựa chọn nhà cung cấp carton theo những tiêu chí nào; và nhà cung cấp nào đáp ứng yêu cầu để PVM sản xuất sản phẩm cung cấp cho thị trường xuất khẩu.

Bảng câu hỏi khảo sát bán cấu trúc đã gửi và thảo luận với giám đốc chuỗi cung ứng, trưởng phòng thu mua và giám sát thu mua công ty PVM nhằm xác định sơ bộ những tiêu chí quan trọng trong việc lựa chọn nhà cung cấp carton cho công ty PVM. Kết quả phản hồi ý kiến của 3 chuyên gia trên được tổng hợp lại và phỏng vấn tay đôi với trưởng phòng thu mua của công ty $\mathrm{PVM}$, chuyên gia có thâm niên công tác tại công ty PVM 15 năm và từng làm việc tại nhiều công ty có tầm cỡ trong lĩnh vực thực phẩm ở vị trí mua hàng. Mục đích nhằm xác định các tiêu chí chính và phụ quan trọng và cần thiết nhất đối với PVM khi chọn nhà cung cấp carton.

\section{KẾT QUẢ VÀ THẢO LUẬN}

\subsection{Các tiêu chí để lụa chọn nhà cung cấp trong} quản lý chuỗi cung úng

\subsubsection{Tóm tắt các tiêu chí để lựa chọn nhà cung cấp dụa trên các nghiên cứu truớc.}

Hiện nay, các tiêu chí đánh giá nhà cung cấp từ các nghiên cứu trước được kế thừa và cải tiến cho phù hợp với bối cảnh hội nhập và toàn cầu hoá. Dickson khảo sát 273 người trưởng phòng mua hàng và xếp hạng 23 tiêu chí theo mức độ quan trọng trong quá trình đánh giá và lựa chọn nhà cung ứng [7]. Weber và cộng sự tiến hành khảo sát lại những tiêu chí đánh giá này và mức độ quan trọng của từng tiêu chí có thay đổi so với nghiên cứu của Dickson [1]. Một số tình huống 
nghiên cứu chỉ sử dụng 9 tiêu chí chính [2] hoặc 5 tiêu chí [3] khi lựa nhà cung cấp. Trong bối cảnh toàn cầu hóa, một số những tiêu chí mới bổ sung cho việc lựa chọn nhà cung cấp phù hợp: tiêu chí độ tin cậy, độ linh động, trách nhiệm môi trường, xã hội, khả năng đáp ứng JIT,...Thiruchelvam và Tookey phát triển 36 tiêu chí, có kế thừa 23 tiêu chí của Dickson [12].

Nhìn chung, tiêu chí đánh giá nhà cung cấp có thể được phân thành 2 loại: định tính và định lượng. Sự lựa chọn tiêu chí nào còn tùy thuộc vào tình trạng của từng công ty. Tuy nhiên, một số tiêu chí cơ bản như giá cả, chất lượng và tình trạng giao hàng là những tiêu chí thường được sử dụng rộng rãi.
3.1.2.Xây dụng các tiêu chí chính và tiêu chí phụ cho lụa chọn nhà cung cấp carton trong quản lý chuỗi cung úng của công ty PVM

Kết quả phỏng vấn giám đốc chuỗi cung ứng, trưởng phòng thu mua và giám sát thu mua công ty PVM tìm ra 13 tiêu chí quan trọng: chất lượng, giao hàng, năng lực sản xuất, giá cả, khả năng kỹ thuật, tình hình tài chính, quản lý và tổ chức, JIT, dịch vụ sửa chữa, vị trí địa lý, độ tin cậy, độ linh hoạt, trách nhiệm môi trường. Kết quả phỏng vấn tay đôi với chuyên gia công ty PVM với dàn bài phỏng vấn chi tiết, tìm được 6 tiêu chí chính và các tiêu chí phụ của từng tiêu chí chính quan trọng và cần thiết nhất đối với PVM khi lựa chọn nhà cung cấp carton (Bảng 2).

Bảng 2: Tiêu chí lựa chọn nhà cung cấp cartontrong quản lý chuỗi cung ứng của công ty PVM

\begin{tabular}{|c|c|}
\hline $\begin{array}{l}\text { Tiêu chí } \\
\text { chính }\end{array}$ & Tiêu chí phụ \\
\hline \multirow{2}{*}{ (1) Chất lượng } & $\begin{array}{l}\text { - Chất lượng sản phẩm: đặc trưng sản phẩm, độ bền sản phẩm, shelf-life của sản phẩm, màu } \\
\text { sắc, độ nén, số lần giao hàng bị lồi. }\end{array}$ \\
\hline & - Chất lượng dây chuyền sản xuất: dây chuyền, công nghệ sản xuất. \\
\hline \multirow[b]{2}{*}{ (2) Chi phí } & - Chi phí trực tiếp: giá bán. \\
\hline & - Chi phí gián tiếp: chi phí làm trục in, bản in, chi phí giao hàng, chi phí làm mẫu. \\
\hline \multirow{2}{*}{ (3) Giao hàng } & $\begin{array}{l}\text { - Thời gian giao hàng: khoảng thời gian giao hàng (lead-time), \% giao hàng trế, thời gian giữ } \\
\text { tồn kho. }\end{array}$ \\
\hline & - Số lượng giao hàng: số lượng đặt hàng tối thiểu, \% giao hàng thiếu số lượng. \\
\hline \multirow{3}{*}{ (4) Độ tin cậy } & - Quản lý và tố chức: chứng chỉ ISO, quy trình kiếm soát chât lượng. \\
\hline & - Máy móc, năng lực sản xuất và nhà xưởng: số lượng máy móc, diện tích nhà xưởng. \\
\hline & - Lịch sử công ty: lịch sử hoạt động, danh tiếng của công ty trên thị trường. \\
\hline \multirow{2}{*}{ (5) Tài chính } & - Tài chính của nhà máy: độ ôn định tài chính, lịch sử tài chính, vốn điều lệ. \\
\hline & - Tài chính của sản phẩm:doanh thu bán hàng của sản phẩm. \\
\hline \multirow{2}{*}{ (6) Dịch vụ } & $\begin{array}{l}\text { - Hô̂ trợ kỹ thuật: xử lý sự cố, hố trợ vân đề kỹ thuật, chất lượng, hồ trợ, tư vấn thiêt kê sản } \\
\text { phẩm. }\end{array}$ \\
\hline & $\begin{array}{l}\text { - Phản hồi thông tin: độ chính xác của thông tin, mức độ chuyên nghiệp trong công việc, thời } \\
\text { gian phản hồi thông tin. }\end{array}$ \\
\hline
\end{tabular}

\section{Trang 138}


3.2.Áp dụng phương pháp AHP lựa chọn nhà cung cấp carton cho công ty bánh kẹo PVM

\subsubsection{Quy trình nghiên cứu lụa chọn nhà cung} cấp theo phương pháp AHP

Công ty PVM có sẵn một nhà cung cấp hiện tại đang cung cấp carton cho thị trường nội địa là công ty $\mathrm{BH}$ và công ty đang đánh giá đưa phương án để phân tích thay thế khi mở rộng thị trường nước ngoài. Quá trình lựa chọn nhà cung cấp gồm giai đoạn: sơ bộ và AHP. Ở giai đoạn sơ bộ, dựa trên những thông tin từ website, ý kiến chuyên gia (giám đốc chuỗi cung ứng và giám sát kỹ thuật của PVM), một số nhà cung cấp carton uy tín: công ty TA; công ty TVL; công ty OVN; công ty ALM. Tiêu chí lựa chọn sơ bộ: gần nhà máy PVM, thuộc tập đoàn lớn, thích ứng nhanh với tình hình công PVM. Công ty TA và $\mathrm{ALM}$ thuộc sở hữu tập đoàn SCG, nhưngTA gần công ty $\mathrm{PVM}$ hơn. TA đã từng gửi mẫu sản phẩm từ đầu năm 2014 và được PVM thử nghiệm chất lượng một số sản phẩm TA đáp ứng được những yêu cầu của PVM. Vì vậy, TA được chọn để đưa vào phân tích thay thế.

Quy trình đánh giá và lựa chọn nhà cung cấp theo phương pháp AHP đề xuất cho công ty PVM như Hình 1.

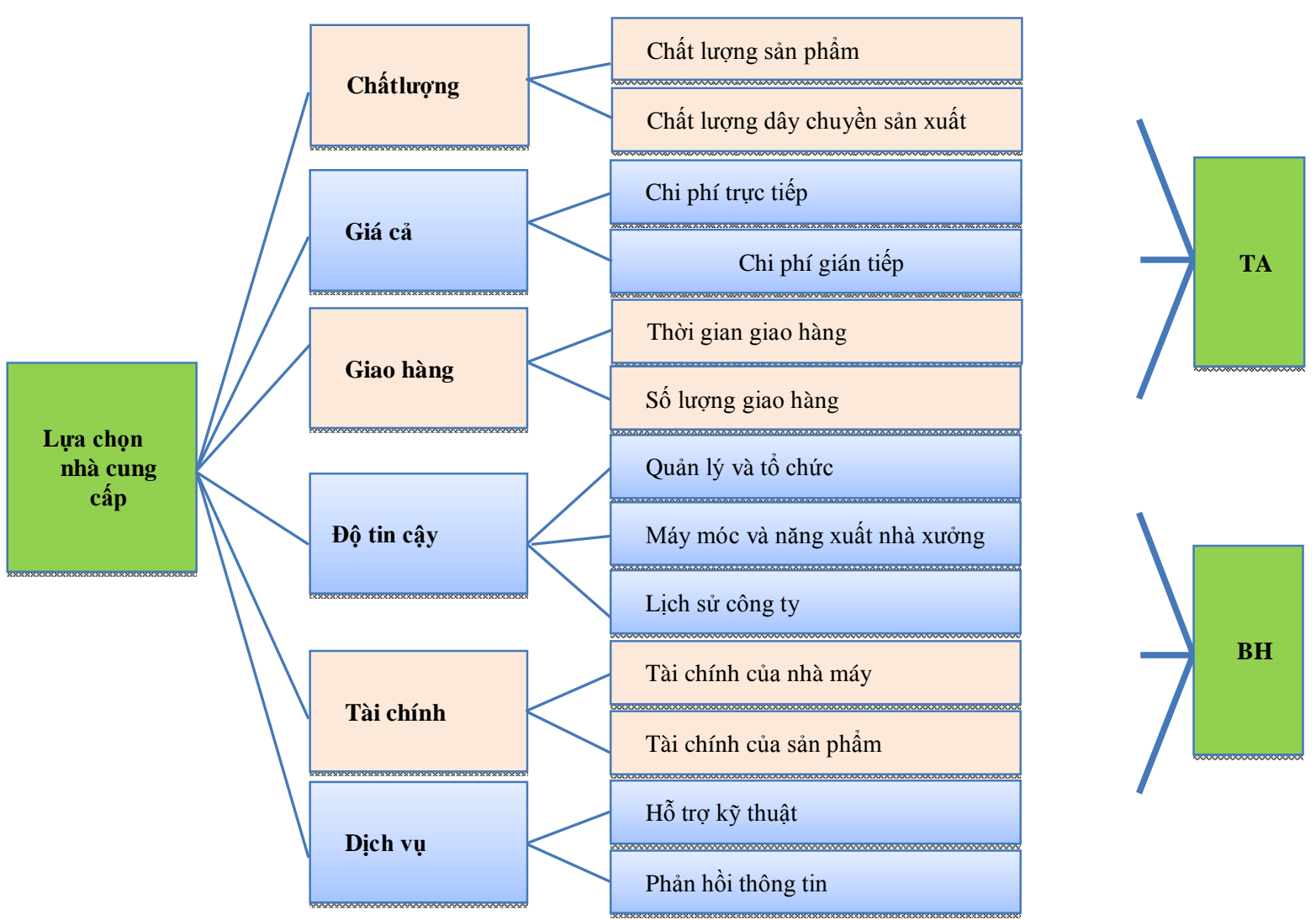

Hình 1: Quy trình đánh giá và lựa chọn nhà cung cấp theo phương pháp AHP đề xuất cho công ty PVM 


\subsubsection{Phân tích và so sánh giữa các phưong án}

\section{Khảo sát dữ liệu cho AHP}

a. Thu thập đánh giá mức độ ưu tiên cho các tiêu chí chính
Kết quả của mức độ ưu tiên của các tiêu chính được trình bày ở Bảng 3 và kết quả ma trận trọng số giữa các tiêu chí chính (Bảng 4). Thang đo đánh giá được sử dụng trong quá trình thu thập này là thang đo từ 1 đến 9 .

Bảng3: Ma trận so sánh mức độ quan trọng giữa các tiêu chí chính

\begin{tabular}{l|c|c|c|c|c|c}
\hline & Chất lượng & Chi phí & Giao hàng & Độ tin cậy & Tài chính & Dịch vụ \\
\hline Chất lượng & 1 & 1 & 5 & 5 & 6 & 6 \\
\hline Chi phí & 1 & 1 & 5 & 5 & 5 \\
\hline Giao hàng & $1 / 5$ & $1 / 5$ & 1 & 5 & 6 \\
\hline Độ tin cậy & $1 / 5$ & $1 / 5$ & $1 / 5$ & 1 & 6 \\
\hline Tài chính & $1 / 6$ & $1 / 5$ & $1 / 6$ & $1 / 2$ & 1 & 1 \\
\hline Dịch vụ & $1 / 6$ & $1 / 4$ & $1 / 6$ & 1 & 1 & 1 \\
\hline Tống & 2.7333 & 2.850 & 11.533 & 17.500 & 21.000 & 19.000 \\
\hline
\end{tabular}

Bảng 4: Ma trận trọng số giữa các tiêu chí chính

\begin{tabular}{|c|c|c|c|c|c|c|c|c|c|}
\hline & $\begin{array}{l}\text { Chất } \\
\text { lượng }\end{array}$ & $\begin{array}{l}\text { Chi } \\
\text { phí }\end{array}$ & $\begin{array}{l}\text { Giao } \\
\text { hàng }\end{array}$ & $\begin{array}{l}\text { Độ tin } \\
\text { cậy }\end{array}$ & Tài chính & $\begin{array}{l}\text { Dịch } \\
\text { vu }\end{array}$ & $\begin{array}{c}\text { Trọng số } \\
\text { (Trung bình dòng) }\end{array}$ & $\begin{array}{l}\text { Tổng của } \\
\text { dòng }\end{array}$ & $\begin{array}{c}\text { Tổng } \\
\text { chuẩn hóa }\end{array}$ \\
\hline $\begin{array}{l}\text { Chất } \\
\text { lượng }\end{array}$ & 0.366 & 0.351 & 0.434 & 0.286 & 0.286 & 0.316 & 0.340 & 2.484 & 7.3163 \\
\hline Chi phí & 0.366 & 0.351 & 0.434 & 0.286 & 0.238 & 0.211 & 0.314 & 2.332 & 7.4240 \\
\hline Giao hàng & 0.073 & 0.070 & 0.087 & 0.286 & 0.286 & 0.316 & 0.186 & 1.217 & 6.5338 \\
\hline Độ tin cậy & 0.073 & 0.070 & 0.017 & 0.057 & 0.095 & 0.053 & 0.061 & 0.374 & 6.1333 \\
\hline Tài chính & 0.061 & 0.070 & 0.014 & 0.029 & 0.048 & 0.053 & 0.046 & 0.280 & 6.1238 \\
\hline Dịch vụ & 0.061 & 0.088 & 0.014 & 0.057 & 0.048 & 0.053 & 0.053 & 0.326 & 6.1072 \\
\hline Tổng & 1 & 1 & 1 & 1 & 1 & 1 & 1 & & 39.638 \\
\hline
\end{tabular}

Để phân tích độ nhất quán của ma trận trọng số giữa các tiêu chí chính, tỷ số nhất quán $\mathrm{CR}$ được tính theo công thức: $\mathrm{CR}=\mathrm{CI} / \mathrm{RI}$; $\mathrm{CI}=$ $(\lambda \max -n) /(n-1)$. Dựa vào công thức ta có $\mathrm{CI}$ như sau: $\mathrm{CI}=(39.638-6) /(6-1)=0.121$; do đó, $\mathrm{CR}=\mathrm{CI} / \mathrm{RI}=0.097<0.1$ (ứngvới $\mathrm{n}=6$ ta có $\mathrm{RI}$ là 1.24). Do CR < $10 \%$ nên bộ trọng số của tiêu chí chính đảm bảo tính nhất quán. Dũ liệu này có thể sử dụng để thực hiện cho việc đánh giá theo phương pháp AHP. b. Thu thập đánh giá mức độ ưu tiên cho các tiêu chí phụ

Sau khi thu thập dữ liệu về so sánh mức độ ưu tiên của các tiêu chí phụ, bộ ma trận so sánh mức độ quan trọng giữa các cặp tiêu chí phụ như bên dưới (Bảng 5).

\section{Trang 140}


Bảng 5: Bộ ma trận so sánh mức độ quan trọng giữa các cặp tiêu chí phụ

\begin{tabular}{|c|c|c|c|c|c|c|}
\hline Chất lượng & $\begin{array}{l}\text { Chất lượng } \\
\text { sản phẩm }\end{array}$ & $\begin{array}{l}\text { Chất lượng dây } \\
\text { chuyền sản } \\
\text { xuất }\end{array}$ & & Chi phí & $\begin{array}{l}\text { Chi phí trực } \\
\text { tiếp }\end{array}$ & $\begin{array}{l}\text { Chi phí gián } \\
\text { tiếp }\end{array}$ \\
\hline \begin{tabular}{|lll}
$\begin{array}{l}\text { Chất } \\
\text { phẩm }\end{array}$ & lượng & sản \\
\end{tabular} & 1 & 5 & & Chi phí trực tiếp & 1 & 1 \\
\hline $\begin{array}{l}\text { Chất lượng dây } \\
\text { chuyền sản xuất }\end{array}$ & $1 / 5$ & 1 & & Chi phí gián tiếp & 1 & 1 \\
\hline Giao hàng & $\begin{array}{l}\text { Thời gian } \\
\text { giao hàng }\end{array}$ & $\begin{array}{l}\text { Số lượng giao } \\
\text { hàng }\end{array}$ & & Tài chính & $\begin{array}{lr}\text { Tài } & \text { chính } \\
\text { của } & \text { nhà } \\
\text { máy } & \\
\end{array}$ & 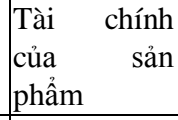 \\
\hline Thời gian giao hàng & 1 & 5 & & $\begin{array}{l}\text { Tài chính của nhà } \\
\text { máy }\end{array}$ & 1 & 1 \\
\hline Số lượng giao hàng & $1 / 5$ & 1 & & $\begin{array}{l}\text { Tài chính của sản } \\
\text { phẩm }\end{array}$ & 1 & 1 \\
\hline Độ tin cậy & $\begin{array}{l}\text { Quản lý và } \\
\text { tồ chức }\end{array}$ & $\begin{array}{l}\text { Máy móc, nhà } \\
\text { xưởng }\end{array}$ & $\begin{array}{l}\text { Lịch sử } \\
\text { công ty }\end{array}$ & Dịch vụ & $\begin{array}{l}\text { Hỗ trợ kỹ } \\
\text { thuật }\end{array}$ & $\begin{array}{l}\text { Phản hồi } \\
\text { thông tin }\end{array}$ \\
\hline Quản lý và tổ chức & 1 & 1 & 5 & Hỗ trợ kỹ thuật & 1 & 1 \\
\hline \begin{tabular}{|l}
$\begin{array}{l}\text { Máy móc, nhà } \\
\text { xưởng }\end{array}$ \\
\end{tabular} & 1 & 1 & 5 & Phản hồi thông tin & 1 & 1 \\
\hline Lịch sử công ty & $1 / 5$ & $1 / 5$ & 1 & & & \\
\hline
\end{tabular}

\subsection{Kết quả xử lí số liệu bằng phần mềm Expert phương pháp AHP được thực hiện với sự hỗ trợ}

\section{Choice}

Quá trình tính toán mô hình đánh giá theo củaphần mềm Expert Choice 11.0. Kết quả được được trình bày ở Bảng 6, Bảng 7 và Hình 2 .

Bảng 6: Các trọng số của các tiêu chí và các phương án thay thế

\begin{tabular}{|c|c|c|c|c|c|c|c|c|}
\hline \multirow{2}{*}{\multicolumn{5}{|c|}{ Tiêu chí }} & \multicolumn{4}{|c|}{ Nhà cung cấp } \\
\hline & & & & & \multicolumn{2}{|c|}{$\mathrm{BH}$} & \multicolumn{2}{|l|}{ TA } \\
\hline $\begin{array}{l}\text { Tiêu chí } \\
\text { chính }\end{array}$ & $\begin{array}{l}\text { Trọng } \\
\text { số } \\
\text { cục bộ }\end{array}$ & Tiêu chí phụ & $\begin{array}{l}\text { Trọng số } \\
\text { cục bộ }\end{array}$ & $\begin{array}{l}\text { Trọng số } \\
\text { toàn cục }\end{array}$ & $\begin{array}{l}\text { Trọng số } \\
\text { cục bộ }\end{array}$ & $\begin{array}{l}\text { Trọng số } \\
\text { toàn cục }\end{array}$ & $\begin{array}{l}\text { Trọng số } \\
\text { cục bộ }\end{array}$ & $\begin{array}{l}\text { Trọng số } \\
\text { toàn cục }\end{array}$ \\
\hline \multirow[b]{2}{*}{$\begin{array}{l}\text { Chất } \\
\text { lượng }\end{array}$} & \multirow[b]{2}{*}{0.355} & Chất lượng sản phẩm & 0.833 & 0.296 & 0.500 & 0.148 & 0.500 & 0.148 \\
\hline & & $\begin{array}{l}\text { Chất lượng dây } \\
\text { chuyền sản xuất }\end{array}$ & 0.167 & 0.059 & 0.875 & 0.052 & 0.125 & 0.007 \\
\hline \multirow{2}{*}{ Chi phí } & \multirow{2}{*}{0.334} & Chi phí trực tiếp & 0.500 & 0.167 & 0.250 & 0.042 & 0.750 & 0.125 \\
\hline & & Chi phí gián tiếp & 0.500 & 0.167 & 0.875 & 0.146 & 0.125 & 0.021 \\
\hline \multirow{2}{*}{$\begin{array}{l}\text { Giao } \\
\text { hàng }\end{array}$} & \multirow{2}{*}{0.169} & Thời gian giao hàng & 0.833 & 0.140 & 0.750 & 0.105 & 0.250 & 0.035 \\
\hline & & Số lượng giao hàng & 0.167 & 0.028 & 0.500 & 0.014 & 0.500 & 0.014 \\
\hline \multirow{3}{*}{$\begin{array}{l}\text { Độ } \quad \text { tin } \\
\text { cậy }\end{array}$} & \multirow{3}{*}{0.054} & Quản lý và tổ chức & 0.455 & 0.025 & 0.500 & 0.012 & 0.500 & 0.012 \\
\hline & & Máy móc, nhà xướng & 0.455 & 0.025 & 0.750 & 0.018 & 0.250 & 0.006 \\
\hline & & Lịch sử công ty & 0.091 & 0.005 & 0.875 & 0.004 & 0.125 & 0.001 \\
\hline \multirow{2}{*}{ Tài chính } & \multirow{2}{*}{0.041} & $\begin{array}{l}\text { Tài chính của nhà } \\
\text { máy }\end{array}$ & 0.500 & 0.021 & 0.875 & 0.018 & 0.125 & 0.003 \\
\hline & & $\begin{array}{l}\text { Tài chính của sản } \\
\text { phẩm }\end{array}$ & 0.500 & 0.021 & 0.875 & 0.018 & 0.125 & 0.003 \\
\hline \multirow{2}{*}{ Dịch vụ } & \multirow{2}{*}{0.048} & Hỗ trợ kỹ thuật & 0.500 & 0.024 & 0.500 & 0.012 & 0.500 & 0.012 \\
\hline & & Phản hồi thông tin & 0.500 & 0.024 & 0.500 & 0.012 & 0.500 & 0.012 \\
\hline Tổng & 1.001 & & & 1.002 & & 0.601 & & 0.399 \\
\hline
\end{tabular}


Bảng 7: Kết quả tính toán trọng số của các phương án thay thế

\begin{tabular}{|c|c|c|c|c|c|c|}
\cline { 2 - 6 } \multicolumn{1}{c|}{} & Chất lượng & Chi phí & Giao hàng & Độ tin cậy & Tài chính & Dịch vụ \\
\hline BH & 0.563 & 0.563 & 0.708 & 0.648 & 0.875 & 0.500 \\
\hline TA & 0.437 & 0.438 & 0.292 & 0.353 & 0.125 & 0.500 \\
\hline Tổng cộng & 1.000 & 1.001 & 1.000 & 1.001 & 1.000 & 1.000 \\
\hline
\end{tabular}

Kết quả mô tả trực quan cho mức độ ưu tiên giữa hai phương án thay thế xét cho từng tiêu chí (Hình 2)

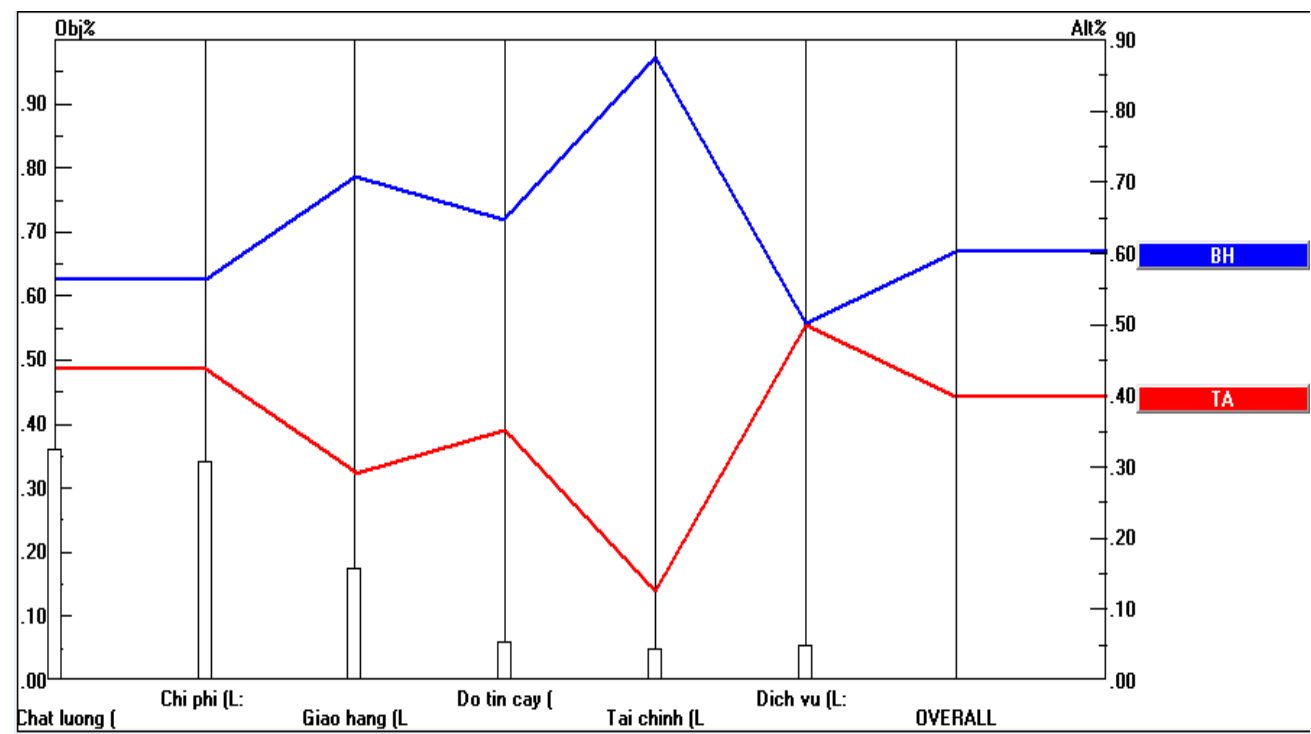

Hình 2: Kết quả phân tích AHP cho các phương án thay thế

Từ kết quả trọng số của các tiêu chí chất lượng, chi phí, giao hàng, độ tin cậy, dịch vụ và tài chính lần lượt là $0.355 ; 0.334 ; 0.169 ; 0.054$; 0.048; 0.041 (Bảng 5), việc lựa chọn nhà cung cấp đối với công ty PVM ưu tiên theo thứ tự tiêu chí: Chất lượng $>$ Chi phí $>$ Giao hàng $>$ Độ tin cậy $>$ Dịch vụ $>$ Tài chính. Từ kết quả tính mức độ ưu tiên giữa công ty $\mathrm{BH}$ và công ty $\mathrm{TA}$ theo các tiêu chí (Bảng 6), công ty BH có ưu thế hơn công ty TA. Kết quả trọng số toàn cục cuối cùng xét cho tất cả các tiêu chí của công ty $\mathrm{BH}$ và $\mathrm{TA}$ lần lượt là 0.601 và 0.399 (Hình 2) cho thấy công ty BH có ưu thế hơn công ty TA. Vì vậy, công ty $\mathrm{BH}$ được đề xuất là nhà cung cấp carton cho công ty PVM phát triển thêm ở thị trường xuất khẩu. Việc chọn BH giúp PVM tiết kiệm thời gian đánh giá, thẩm định và huấn luyện nhà cung cấp. Tuy nhiên, BH đang là nhà cung cấp carton chính ở mảng thị trường nội địa, nên khi phát triển $\mathrm{BH}$ cho thị trường xuất khẩu có thể dẫn tới việc quá tải; PVM nên có chiến lược phù hợp.

\section{KẾT LUẬN VÀ KIẾN NGH!}

Nghiên cứu này đã: (a) lý luận sự phù hợp của việc áp dụng phương pháp AHP để giải bài toán ra quyết định đa tiêu chí theo cách phân tích thứ bậc và tổng hợp các tiêu chí quan trọng lựa chọn nhà cung cấp trong quản lý chuỗi cung ứng về mặt lý thuyết; và (b) tìm các tiêu chí đánh giá

Trang 142 
lựa chọn nhà cung cấp carton cho công ty PVM theo phương pháp AHP. Kết quả giúp công ty PVM nhìn đúng đắn và toàn diện hơn về nhà cung ứng.

Việc xác định tiêu chí lựa chọn nhà cung ứng và phương pháp đánh giá ra quyết định lựa chọn là hai giai đoạn quan trọng và sẽ có thể thay đổi tùy vào mục tiêu và ngành nghề công ty.

\section{TÀI LIỆU THAM KHẢO}

[1]. C. A.Weber, J. R. Current, W.C. Benton, Vendor Selection Criteria and Methods, European Journal of Operational Research, 50(1), 2-18(1991).

[2]. C. Muralidharan, N. Anantharaman, S.G. Deshmukh, A Multi-Criteria Group Decisionmaking Model for Supplier Rating, Journal of Supply Chain Management, 38(3), 22-33 (2002).

[3]. C.C. Li, Y.P. Fun, J.S. Hung, A new measure for supplier performance evaluation, IIE Transactions, 29(9), 753-758 (1997).

[4]. D. Asamoah, J. Annan, S. Nyarko, AHP Approach for Supplier Evaluation and Selection in a Pharmaceutical Manufacturing Firm in Ghana, International Journal of Business and Management, 7(10), 49-62 (2012).

[5]. D. S. Verma, A. Pateriya, Supplier Selection through Analytical Hierarchy Process: A Case Study In Small Scale Manufacturing Organization, International Journal of Engineering Trends and Technology, 45(5), 1428-1433, (2013).

[6]. F. Tahriri, M.R. Osman, A. Ali, R.M. Yusuff, A. Esfandiary, AHP approach for supplier evaluation and selection in a steel manufacturing company, Journal of Industrial Engineering and Management, 1(2), 54-76 (2013).

[7]. G.W. Dickson, An Analysis of Vendor Selection Systems and Decisions,Journal of Purchasing, 2(1), 5-17 (1966).
Phương pháp AHP trong nghiên cứu này được áp dụng cho bài toán lựa chọn nhiều nhà cung cấp cho một loại sản phẩm, có thể áp dụng cho các vấn đề tương tự ở các công ty khác nhau. Ngoài ra, hướng nghiên cứu này có thể mở ra cho việc chọn nhiều nhà cung cấp cho nhiều sản phẩm với kỹ thuật Fuzzy, Dematel và nên lượng hoá tính không chắc chắn của thông tin.

[8]. H. Jin, C. L. Liu, X. Y. Wang, AHP Approach for Supplier Selection in a Polyamide Fiber Plant in Supply Chain Management, Advanced Materials Research, 299-300, 1252-1255 (2011).

[9]. M. Velasquez, P. T. Hester, An Analysis of Multi-Criteria Decision Making Methods (Norfolk, VA, USA, 2013).

[10].P. T. Trương, Khoá luận Thạc Sỹ, Đánh giá và lựa chọn nhà cung cấp tại công ty TNHH May Việt Lê, Trường Đại Học Bách Khoa, TP.HCM (2012).

[11].S. Kumar, N. Parashar, A. Haleem, Analytical Hierarchy Process Applied to Vendor Selection Problem: Small Scale, Medium Scale and Large Scale Industries, Business Intelligence Journal 2(2), 355-362 (2009).

[12].S.Thiruchelvam, J.E. Tookey, Evolving trends of supplier selection criteria and methods,International Journal of Automotive and Mechanical Engineering, 4, 437-454 (2011).

[13].T. H. N. Vo, Khoá luận Thạc Sỹ, Lựa chọn nhà cung cấp thiết bị di động viễn thông tại công ty mạng lưới Viettel: một hướng tiếp cận dùng AHP, Trường Đại Học Bách Khoa, TP.HCM (2015).

[14].T. L. Saaty, The Analytical Hierarchy Process(McGraw-Hill, New York, 1980).

[15].Z. Degraeve, F. Roodhoft, Actively Selecting Suppliers Using Total Cost of Ownership, Journal of Supply Chain Management, 35(1), 5-10(1999). 